\title{
Deep ISOPHOT far-infrared imaging of M 86^
}

\author{
M. Stickel ${ }^{1}$, J. N. Bregman ${ }^{2}$, A. C. Fabian ${ }^{3}$, D. A. White ${ }^{3}$, and D. M. Elmegreen ${ }^{4}$ \\ 1 Max-Planck-Institut für Astronomie, Königstuhl 17, 69117 Heidelberg, Germany \\ 2 Dept. of Astronomy, University of Michigan, Ann Arbor, MI 48109-1090, USA \\ 3 University of Cambridge, Institute of Astronomy, Madingly Road, Cambridge CB30HA, UK \\ ${ }^{4}$ Dept. of Physics and Astronomy, Vassar College, Poughkeepsie, NY 12604, USA
}

Received 5 June 2002 / Accepted 24 September 2002

\begin{abstract}
Deep far-infrared (FIR) imaging data obtained with ISOPHOT at $60 \mu \mathrm{m}, 90 \mu \mathrm{m}, 150 \mu \mathrm{m}$, and $180 \mu \mathrm{m}$ reveal a complex FIR morphology of the Virgo cluster elliptical M 86 (NGC 4406). A close pair of sources is associated with the centre of the M 86 galaxy and optically discovered dust streamers. Both components have a cold FIR spectrum from the thermal emission of dust with a temperature of $\approx 18 \mathrm{~K}$. A compact off-center source lying $\approx 3.5^{\prime}$ north-west is likely an unrelated background source. The brightest FIR source long-wards of $90 \mu \mathrm{m}$ consists of a compact central core surrounded by an extended halo. It lies close to the nearby spiral galaxy NGC 4402 at the position of the strong northern depression in $\mathrm{X}$-ray brightness. It represents the first detection of an intergalactic dust cloud in the intergalactic region between galaxies. Additionally, three much fainter FIR sources are detected in the field. The ISOPHOT data do not support the earlier suggestion based on IRAS and X-ray data that the unresolved north-western component is cold dust removed from M 86 by ram pressure stripping. The observational evidence suggests that at least for the overall FIR morphology, gravitational interactions between M 86 and neighboring galaxies are much more important than ram stripping of dust. Moreover, the X-ray morphology appears to be partially affected by dusty foreground absorbers. Since M 86 is not showing signs of ongoing stripping of interstellar dust by the intra-cluster medium, a clear case for this dynamical interaction has yet to be found.
\end{abstract}

Key words. galaxies: individual: M 86 - galaxies: clusters: general - intergalactic medium - infrared: general infrared: galaxies - methods: data analysis

\section{Introduction}

The interaction of the hot X-ray emitting gas in galaxy clusters with the interstellar medium (ISM) of cluster galaxies while moving through the cluster is likely to have a significant influence on the composition and distribution of the ISM and thereby on the galaxy evolution. There are several indirect hints for this ram pressure interaction such as $\mathrm{HI}$ deficient spiral galaxies (e.g. Giovanelli \& Haynes 1983; Solanes et al. 2001), truncated HI disks of spiral galaxies (e.g. Cayatte et al. 1994), and HI trails or clouds (e.g. van Driel \& van Woerden 1989; Irwin \& Sarazin 1996). Although dust is mixed with the interstellar gas and is expected to participate in the interaction, observational statistical evidence is somewhat controversial. Doyon \& Joseph (1989) have found a significant dust stripping

Send offprint requests to: $\mathrm{M}$. Stickel,

e-mail: stickel@mpia-hd.mpg.de

* Based on observations with ISO, an ESA project with instruments funded by ESA Member States (especially the PI countries: France, Germany, The Netherlands and the UK) and with the participation of ISAS and NASA. of spirals near the cluster core, whereas Bicay \& Giovanelli (1987) and Contursi et al. (2001) conclude that the FIR properties and the dust content of most cluster galaxies are not affected by the environment. Moreover, a clear case of dust being stripped from an individual spiral galaxy is currently not known.

In fact, the only case where observational data indicate ongoing dust stripping due to the movement of the galaxy relative to the cluster environment is the bright Virgo elliptical M 86 (NGC 4406). It is a luminous X-ray emitting galaxy (distance $19.5 \mathrm{Mpc}$, Neilsen \& Tsvetanov 2000) with distorted optical isophotes (Nulsen \& Carter 1987; Katsiyannis et al. 1998; Elmegreen et al. 2000) having a rather high negative radial velocity of $-1364 \mathrm{~km} \mathrm{~s}^{-1}$ (Faber et al. 1989) relative to the cluster as a whole. It is likely the dominant member of a small galaxy group entering the Virgo cluster from the back (Binggeli 1999; Schindler et al. 1999).

Early X-ray observations of M 86 showed a region of extended emission (designated the "plume") not coincident with the center of the galaxy, which was interpreted as hot ISM stripped from the galaxy (Forman et al. 1979). A more recent X-ray study of M 86 based on ROSAT PSPC and HRI 
imaging data (Rangarajan et al. 1995) revealed a complex overall X-ray morphology, which together with abundance measurements corroborated the suggestion that the plume is ISM removed in a single blob from M 86 by ram pressure. ASCA data in the soft X-ray band (0.7-4 keV) also clearly showed the asymmetric extended X-ray emission, while the harder (4-7 keV) ASCA X-Ray band is dominated by several compact sources (Matsushita et al. 1994). These observations agree with the structure seen in a higher resolution Chandra image (Forman et al. 2001), which in particular shows the decline in $\mathrm{X}$-ray surface brightness just north of the plume to be quite steep and likely of small angular extent. The Chandra temperature map confirms the rather cool $(T \approx 0.6 \mathrm{kev}) \mathrm{X}$-ray emission of M 86 and the plume and the warmer surrounding Virgo X-ray emission $(T \approx 2 \mathrm{kev})$, originally inferred from ROSAT observations (Rangarajan et al. 1995). Although a three-dimensional simulation of the gas stripping by Toniazzo \& Schindler (2001) reproduced the plume, none of the projections showed a good match to the overall X-ray morphology, indicating that stripping alone is not sufficient to explain the observed X-ray structure. Particularly, the steep northern decline and the adjacent region of decreased X-ray emission (the northern void of Rangarajan et al. 1995) is likely not a Mach cone of the stripping process, but instead requires a different explanation (Toniazzo \& Schindler 2001).

During the initial investigation of the IRAS data to search for FIR emission of elliptical galaxies, M 86 was found to be comprised of two components, one coincident with the optical galaxy, and another $\approx 3.5^{\prime}$ north-west of the galaxy center (Knapp et al. 1989). In a more detailed analysis of the IRAS data (White et al. 1991) the stronger off-center $60 \mu \mathrm{m}$ FIR emission appeared to be close to the extended X-ray emission (the plume) while the weaker $100 \mu \mathrm{m}$ FIR component was closely coincident with the center of the optical galaxy. The positional association of the off-center FIR and X-ray emission was interpreted as dust in the ISM removed from M 86 by ram pressure stripping subsequently being heated and destroyed by sputtering, while the asymmetry in the optical isophotes might be caused by starlight scattered by the stripped dust (White et al. 1991).

Remarkably, M 86 has repeatedly been described as virtually free of dust (e.g. Veron-Cetty \& Veron 1988; van den Bergh \& Pierce 1990; Michard 1999), although already Bregman \& Roberts (1990) reported extended dust features $\approx 100^{\prime \prime}$ east from a private communication by J. L. Tonry. After a first non-detection of dust in the very center of M 86 (Tomita et al. 2000), a central dusty disk has eventually been found by Tran et al. (2001).

Based on HI measurements of M 86, Bregman \& Roberts (1990) suggested as an alternative to the ram pressure stripping picture that the X-ray plume results from either cooling gas or a tidal encounter of M 86 with NGC 4402. New high resolution VLA observations showed that the $\mathrm{HI}$ distribution is highly asymmetric, with the strongest peak south of the optical center and weaker emission towards east and northwest (Li \& van Gorkom 2001). Consistent with the non-central HI emission are the repeated non-detections of CO emission in M 86 (Lees et al. 1991; Wiklind et al. 1995; Braine et al. 1997). In view of its X-ray luminosity, M 86 is an exceptionally weak radio source. For the $6 \mathrm{~cm}$ continuum emission, also only upper limits were obtained (Fabbiano et al. 1989).

The suggestion that gravitational interaction plays a role in M 86 is strongly supported by the deep optical imaging data of Elmegreen et al. (2000), who found dust trails inside the optical halo of M 86. These dust features were interpreted as the result of stripping the ISM of the nucleated dwarf elliptical galaxy VCC 882 (NGC 4406B, VCP 455) while moving along its bound orbit around M 86. Although no emission line have been found in optical spectra of M 86 (Ho et al. 1995), the narrow band imaging data of Döbereiner et al. (1996) showed three faint extended $\mathrm{H}_{\alpha}$ emission line regions, one of which is apparently beginning at the position of VCC 882, supporting the tidal interaction picture. Diffuse optical emission surrounding the M 86/M 84 pair and other nearby Virgo spirals as well as a curved structure reminiscent of a partial outer shell at the north-western end of the M 86 major axis (Arnaboldi et al. 1996) provide very strong evidence for gravitational interaction of M 86 with neighboring galaxies.

The hitherto favored interpretation of the FIR emission of M 86 as an example of ongoing ram pressure stripping of dust still rests on the IRAS data alone. With its much higher sensitivity, higher angular resolution, and increased wavelength coverage FIR observations with the ISOPHOT detector aboard the Infrared Space Observatory (ISO; Kessler et al. 1996) give a much more detailed view of the dust distribution, allow a comparison of the FIR morphology with the structures seen at other wavelengths, and shed new light on the different scenarios proposed for the dynamical state of M 86, particularly the ram pressure dust stripping.

\section{Observations and data reduction}

FIR observations of M 86 were obtained on four occasions in June and July 1996 with the FIR cameras of ISOPHOT (Lemke et al. 1996; Lemke \& Klaas 1999) in P32 chopped mode. The complete data set consists of four rectangular maps covering an area of $\approx 15^{\prime} \times 10^{\prime}$, two of which were obtained with the $3 \times 3$ pixel C100 camera at $60 \mu \mathrm{m}$ and $90 \mu \mathrm{m}$ wavelengths, while the other two were obtained with the $2 \times 2$ pixel C200 camera $(150 \mu \mathrm{m}$ and $180 \mu \mathrm{m})$. Total integration times were $2300 \mathrm{~s}$ and $2000 \mathrm{~s}$ for the $\mathrm{C} 10060 \mu \mathrm{m}$ and $90 \mu \mathrm{m}$ maps, respectively, and $3800 \mathrm{~s}$ for both C200 filters. To put the signals on an absolute level, calibration observations with the Fine Calibration Source of ISOPHOT were taken before and after each map.

The IRAS data already indicated that the emission of the various components present in the M 86 field are well below $1 \mathrm{Jy}$. To push the detection of these weak signals to the limits, the pairwise differences of consecutive ramp read-outs instead of the standard ramp slopes were used to derive the detector signals. This allows a larger distribution to be analyzed, leading to considerably more robust results. Moreover, since the overall cirrus background in the direction of M 86 is weak and flat, the C100 and C200 data showed no drift in the pairwise read-out differences, therefore all read-outs belonging to a given raster and chopper position were considered as a single entity, and 
only one signal was derived from the corresponding pairwise distribution.

To get rid of pairwise differences affected by cosmic ray hits, the robust outlier-insensitive myriad estimator (Kalluri \& Arce 1998) was computed and $20 \%$ of the most deviant signals as measured by the absolute deviation were cut off. This outlier removal is similar to a median absolute deviation trimming, but instead of the initial median, the sample myriad is used to determine the outliers. The sample myriad value in turn is a robust estimator of the mode (most common value) of a distribution but does not require binning of the actual data set, and is easily computed by simply minimizing a particular cost function with a tuning constant set to a small value (for details see Kalluri \& Arce 1998). After rejecting the outliers, the trimmed set of pairwise differences was linearly interpolated to a 10 times finer grid and the value giving the minimum value for the myriad cost function accepted as the final signal for each raster point and chopper elongation of the maps. This interpolation scheme was used as an approximation to a full numerical minimization of the myriad cost function, which would computationally be quite demanding.

The derived detector signals were subsequently corrected for signal dependence on ramp integration times to be consistent with calibration observations (Laureijs et al. 2000), dark-current subtracted, and finally flux calibrated with PIA ${ }^{1}$ version 9.1/Cal $\mathrm{G}$ version 6.0 (Gabriel et al. 1997). For the conversion to an absolute flux level, the observations of the ISOPHOT Fine Calibration Source (FCS) obtained at the beginning and end of each scan in each filter were used. The signals from the two accompanying FCS measurements were linearly interpolated in time to calibrate each sky position separately.

The flux calibrated data streams of the detector pixels in each filter, particularly from the $\mathrm{C} 100$ detector, showed significant differences in the overall levels of up to $30 \%$, most likely coming from inappropriately corrected pixel-to-pixel sensitivities (flat field), which moreover appeared to be time-dependent. If not removed, these varying brightness levels would lead to severe stripping and checker board patterns in the final maps. Therefore, robust filtering techniques were used to smooth the data streams, followed by a time-dependent scaling of each individual data stream to the common mean, and removing any residual time trend with robust low-order polynomial fits. This method has proven to be highly effective in removing pixel-topixel sensitivity and time trends in the data streams. Even an overall tilted cirrus background with an angular scale length of the order of the map size or larger is kept, unless the backand-forth raster data streams were obtained along a direction exactly perpendicular to the background gradient, in general a highly unlikely situation, which moreover can be checked using IRAS maps.

Eventually, a single map for each filter was produced from the flat-fielded flux-calibrated data streams of all pixels in one

1 The ISOPHOT data presented in this paper were reduced using PIA, which is a joint development by the ESA Astrophysics Division and the ISOPHOT Consortium. The ISOPHOT Consortium is led by the Max-Planck-Institute für Astronomie, Heidelberg. step by using the Drizzle mapping method (Hook \& Fruchter 1997) within IRAF$^{2}$, which took into account the pixel sizes and inter-pixel distances of the detectors and the detector rollangles. The final map pixel sizes were tuned to be as small as possible without leaving uncovered holes, resulting in $15^{\prime \prime}$ pixels for the C100 $(60 \mu \mathrm{m}$ and $90 \mu \mathrm{m})$ data and $30^{\prime \prime}$ pixels for the $\mathrm{C} 200(150 \mu \mathrm{m}$ and $180 \mu \mathrm{m})$ data. Due to excessive variability, one of the nine $\mathrm{C} 100$ pixels was discarded from the drizzling process, leading to a funny saw-tooth edge of the one-step $60 \mu \mathrm{m}$ and $90 \mu \mathrm{m}$ maps (Figs. 1 and 3).

The P32 chopped mode of ISOPHOT acquired data along the chopping direction with positional offsets much smaller than the detector pixel size. Together with the raster movement of the ISO telescope, the flux calibrated data streams contain a high redundancy, which was utilized to produce a second set of maps for the four filters. First, for each detector pixel and chopper throw, individual full maps with a common pixel size and map center were made using Drizzle within IRAF from the flat-fielded flux calibrated data streams. This procedure gives 117 individual images for the shorter wavelengths bands $(60 \mu \mathrm{m}$ and $90 \mu \mathrm{m})$ from the $\mathrm{C} 100$ detector, and 28 individual images from the longer wavelengths $\mathrm{C} 200$ bands $(150 \mu \mathrm{m}$ and $180 \mu \mathrm{m}$ ) bands. These individual maps were subsequently stacked, and a final averaged map created from the stack using a min-max outlier rejection scheme. To get a less blocky appearance of the final stack-averaged maps, the individual maps were resampled to a twice finer grid before stacking, rejecting and averaging, with a pixel size of $15^{\prime \prime}$ for all four bands. Since the rejection of pixels from the stack was forced during the stack-averaging, the outer regions of the maps not covered by all individual images of all detector pixels and chopper throws were removed, leading to maps somewhat smaller and with softer edges compared to the one-step drizzle maps. This stack-averaging reduction scheme is quite similar to standard near-infrared data processing and is found to suppress quite effectively small scale detector variability, leading to significantly lower detection limits, as shown below.

Eventually, a ISOPHOT FIR “white light” (60-180 $\mu \mathrm{m})$ image covering the full observed area was produced from the mean of the stack-averaged images of the four bands, this time including also the border regions not covered by all detectors pixels and chopper throws. This map represents the deepest FIR image of the M 86 to date.

\section{Results}

Gray-scale representations of the $60 \mu \mathrm{m}, 90 \mu \mathrm{m}, 150 \mu \mathrm{m}$, and $180 \mu \mathrm{m}$ maps with contours overlaid are shown in Figs. 1-8. A comparison of the results from the one-step drizzling (Figs. 1, $3,5,7$ ) and from the stack-averaging (Figs. 2, 4, 6, 8) of the flux-calibrated data streams immediately demonstrates the increase in depths, particularly in the C100 maps, resulting from the stacking and averaging of a large number of individual

\footnotetext{
2 IRAF is distributed by the National Optical Astronomy Observatories, which are operated by the Association of Universities for Research in Astronomy, Inc., under cooperative agreement with the National Science Foundation.
} 


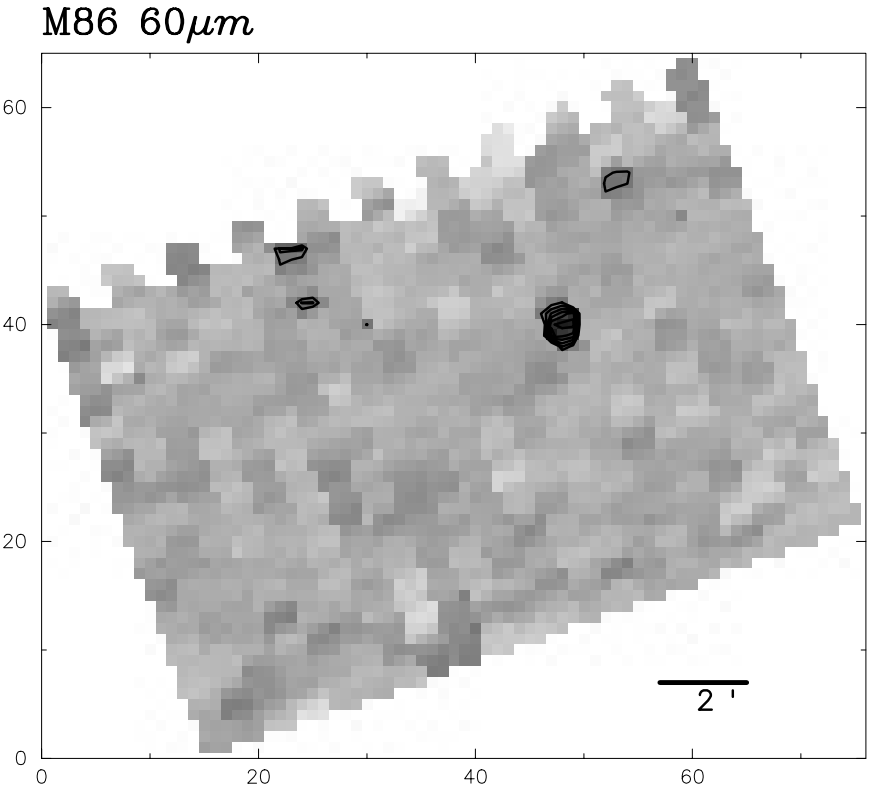

Fig. 1. $60 \mu \mathrm{m}$ map of the field of M 86, obtained by drizzling the complete flux-calibrated ISOPHOT data stream onto the final map output grid with a pixel size of $15^{\prime \prime}$. The saw-tooth upper edge results from discarding one detector pixel due to excessive noise. North is up and east to the left.

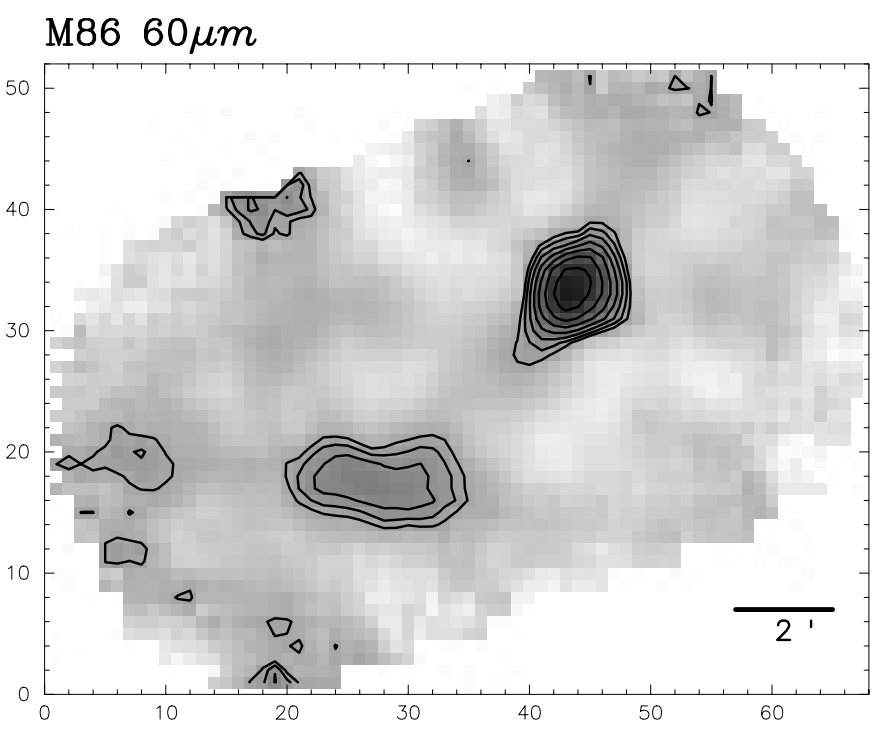

Fig. 2. $60 \mu \mathrm{m}$ map of the field of M 86, obtained by drizzling the fluxcalibrated data stream for each ISOPHOT pixel and chopper position onto a separate output grid, interpolating any uncovered holes, resampling each of the images to a twice finer grid, and averaging the resulting image stack using a forced min-max rejection scheme. Final map pixel size is $15^{\prime \prime}$. North is up and east to the left.

maps. Cross-checks of the positions and fluxes between the one-step and stack-averaged maps were made, and within the uncertainties given by the background and the map pixel size, no systematic deviations were found. Since the stack-averaged maps (Figs. 2, 4, 6, 8) give a much clearer FIR view of the M 86 field, they will mainly be discussed further.

The images reveal an unexpected complex grouping of

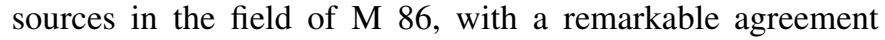

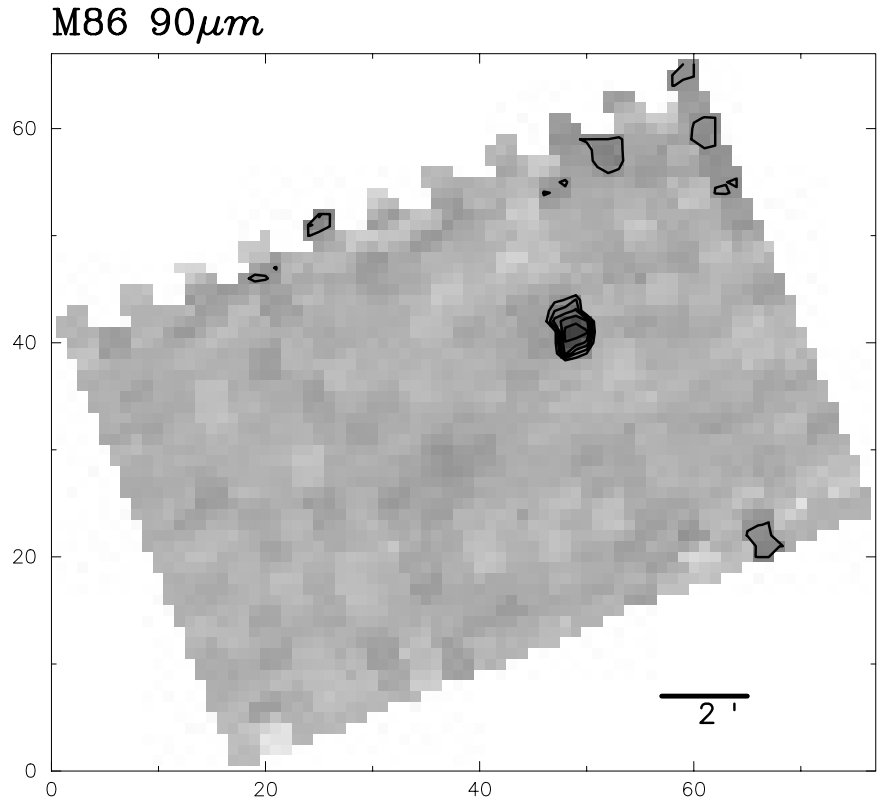

Fig. 3. Same as Fig. 1, but for the $90 \mu \mathrm{m}$ band.

\section{M86 $90 \mu m$}

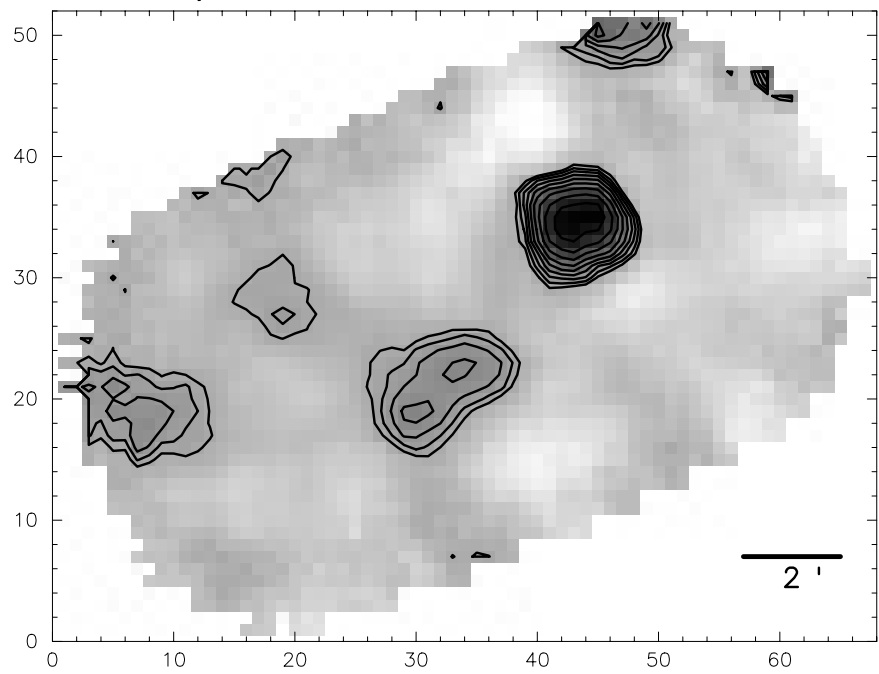

Fig. 4. Same as Fig. 2, but for the $90 \mu \mathrm{m}$ band.

of features across the four wavelength bands. Particularly, the deep FIR "white light" (60-180 $\mu \mathrm{m})$ image (Fig. 9) allows the identification of a total of seven sources in the M 86 area, all of them are lying within the optical stellar halo of the elliptical galaxy. A sketch of the various components apparent in the four bands is shown in Fig. 10 to clarify the arrangement and to indicate the nomenclature of the sources used hereafter.

The brightest source in the $60 \mu \mathrm{m}$ (Fig. 2) and $90 \mu \mathrm{m}$ maps (Fig. 4) is an unresolved point source (M 86-NW) north-west from the map center. At $150 \mu \mathrm{m}$ (Fig. 6), and $180 \mu \mathrm{m}$ (Fig. 8) this compact source clearly decreases in brightness, while a compact double source (M 86/M 86-SE) separated by $\approx 1.5^{\prime}$ and closely coincident with the optical M 86 galaxy is most prominent. It should be noted that the apparent faint bridge in the FIR "white light" $(60-180 \mu \mathrm{m})$ image (Fig. 9) connecting the central pair M 86/M 86-SE with M $86-\mathrm{NW}$ is likely not 


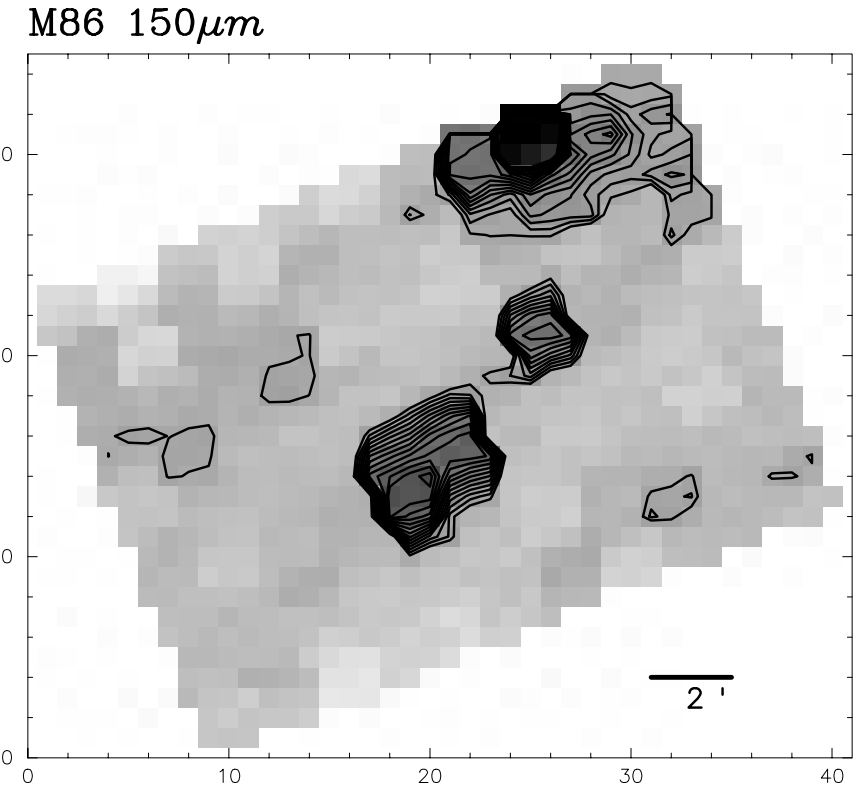

Fig. 5. Same as Fig. 1, but for the $150 \mu \mathrm{m}$ band. Final map pixel size is $30^{\prime \prime}$.

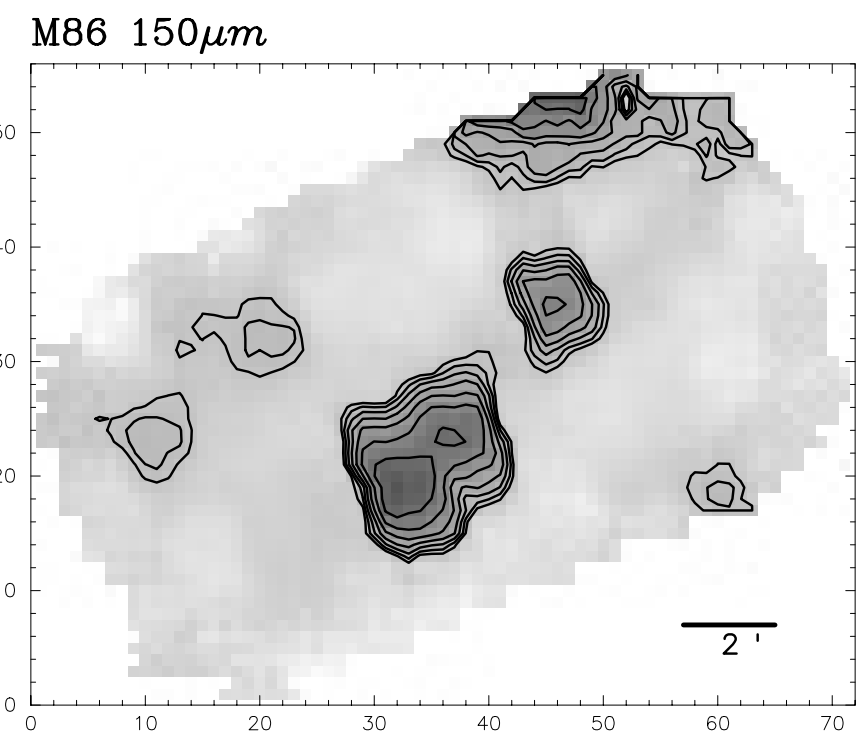

Fig. 6. Same as Fig. 2, but for the $150 \mu \mathrm{m}$ band. Final map pixel size is $15^{\prime \prime}$.

real but due to the overlapping outer wings of the instrumental beam profile.

The M 86/M 86-SE pair is also seen at $90 \mu \mathrm{m}$ (Fig. 4), albeit much fainter. However, at $60 \mu \mathrm{m}$ (Fig. 2), the structure of the pair near the optical position of M 86 appears to be different, lying further to the east at a different position angle. Varying the last step of the map processing using several different rejection schemes and stack averaging methods (mean, median, biweight) resulted in all cases in the same $60 \mu \mathrm{m}$ morphology. This can be taken as evidence that the different appearance is due to a changing source structure at wavelengths below $90 \mu \mathrm{m}$, rather than due to residual variations in the flux-calibrated data streams.

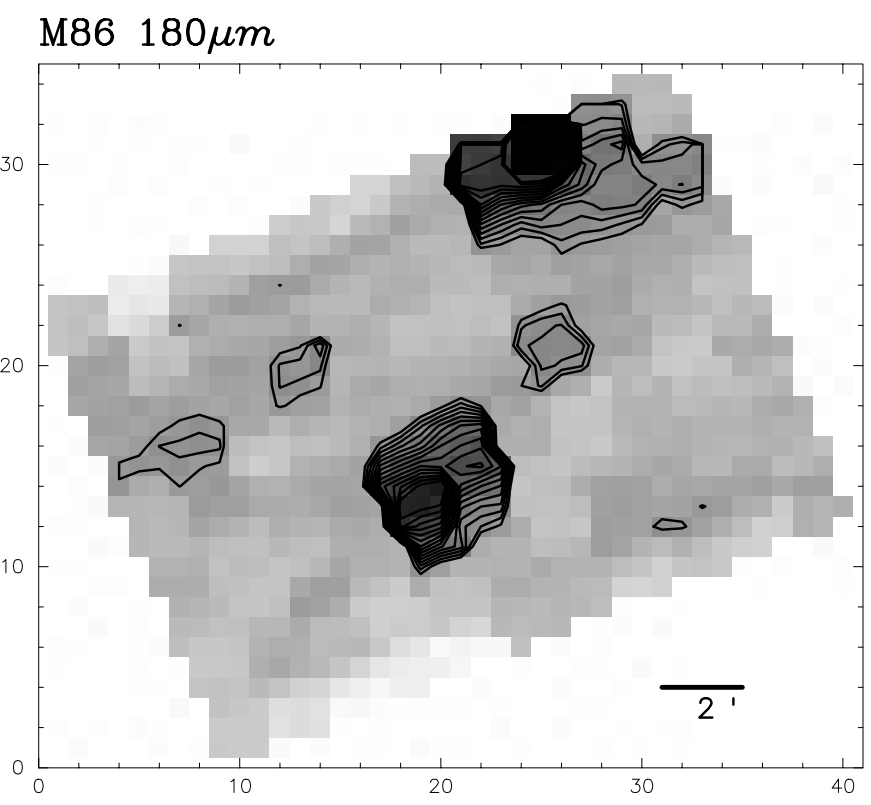

Fig. 7. Same as Fig. 1, but for the $180 \mu \mathrm{m}$ band. Final map pixel size is $30^{\prime \prime}$.

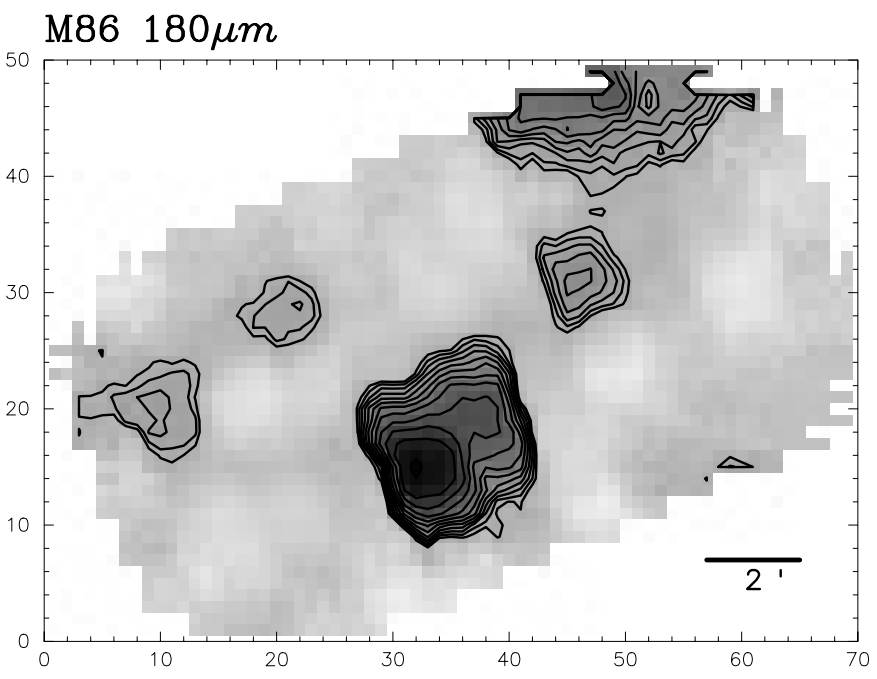

Fig. 8. Same as Fig. 2, but for the $180 \mu \mathrm{m}$ band. Final map pixel size is $15^{\prime \prime}$.

A remarkable source (M 86-FIR 4) appears at the northern edge of the $150 \mu \mathrm{m}$ and $180 \mu \mathrm{m}$ maps (Figs. 5 and 7), which is unfortunately only partially covered by the individual bands. It is most completely seen in the FIR "white light" $(60-180 \mu \mathrm{m})$ image (Fig. 9). Its somewhat irregular morphology consists of a central compact core surrounded by an extended halo elongated roughly along the east-west direction. Its brightness is strongly increasing with wavelength, since it is absent on the $60 \mu \mathrm{m}$ map, only hardly noticeable on the $90 \mu \mathrm{m}$ map, and quite prominent on the $150 \mu \mathrm{m}$ and $180 \mu \mathrm{m}$ maps. It is also not detected in $60 \mu \mathrm{m}$ and $100 \mu \mathrm{m}$ IRAS HIRES maps of the M 86 field obtained from IPAC. This rapidly increasing brightness with increasing wavelength again indicates a rather cold spectral energy distribution (SED), but the partial coverage allows only a somewhat crude photometry. 


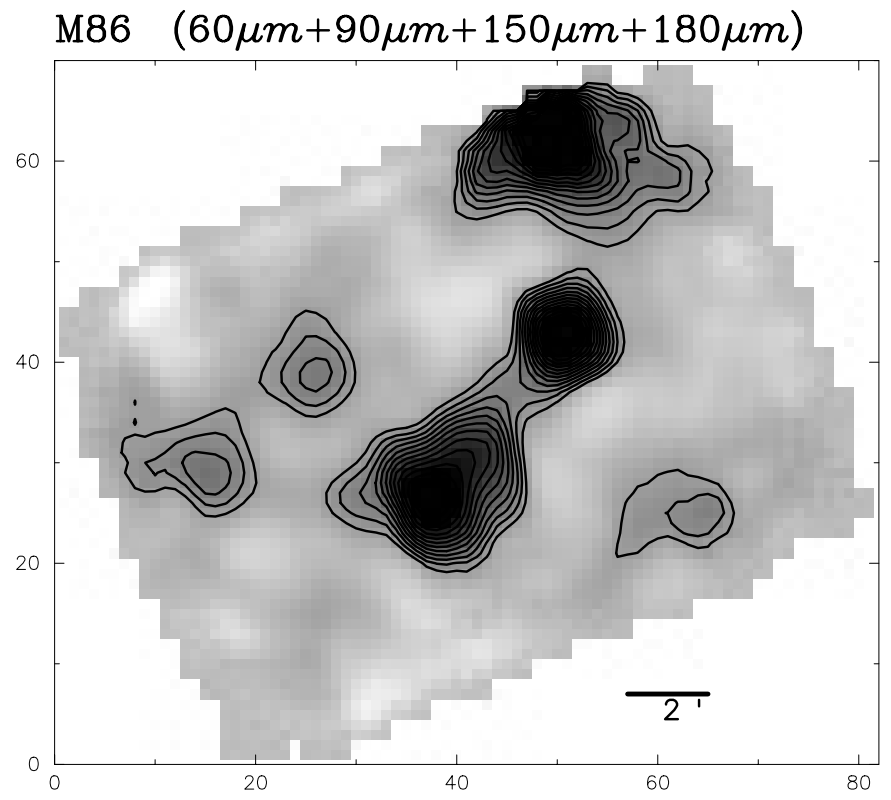

Fig. 9. ISOPHOT FIR “white light" (60-180 $\mu \mathrm{m})$ image of the M 86 field, the mean of the four stack-averaged images including also the border regions. Final map pixel size is $15^{\prime \prime}$.

\section{M86 field (schematic)}

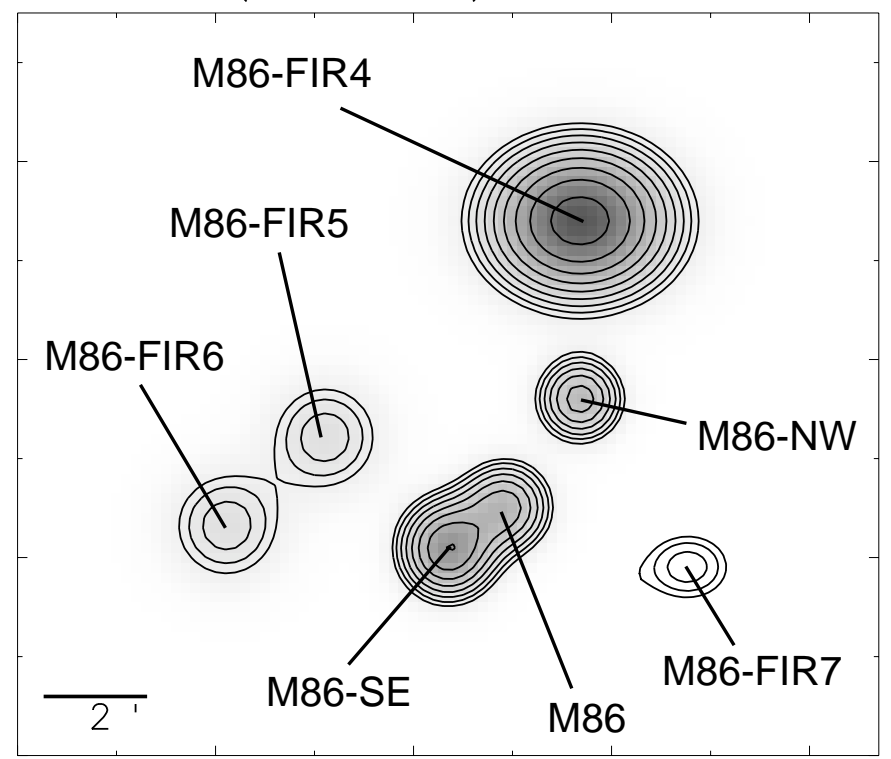

Fig. 10. Composite schematic drawing of all FIR sources in the M 86 field detected by ISOPHOT, based on the FIR "white light" (60-180 $\mu \mathrm{m})$ image. (See also Figs. 1-8 and Table 1.)

Finally, the $90 \mu \mathrm{m}, 150 \mu \mathrm{m}$ and $180 \mu \mathrm{m}$ maps (Figs. 4, 6 and 8) show a pair of vary faint sources (M 86-FIR 5, M 86FIR 6) east of M 86, one component of which (M 86-FIR 5) also appears on the $60 \mu \mathrm{m}$ map (Fig. 1). An even fainter source appears in the co-added FIR "white light" $(60-180 \mu \mathrm{m})$ image $\approx 4^{\prime}$ south-west of the central M 86 source, which is not significant in the four separate stack-averaged maps (Figs. 2, 4, 6 and 8).

Positions and integrated fluxes for the five fully covered sources in the field (except the only partially covered source
M 86-FIR4 and the very faint source M 86-FIR 7) have been measured by fitting two-dimensional Gaussians together with a tilted plane as background to the stack-averaged $60 \mu \mathrm{m}, 90 \mu \mathrm{m}$, $150 \mu \mathrm{m}$, and $180 \mu \mathrm{m}$ maps. The resulting coordinates for the three brighter sources (M 86, M 86-SE, M 86-NW) agree within about one map pixel $\left(15^{\prime \prime}\right)$, while the positions of the diffuse faint pair of sources east of M 86 (M 86-FIR 5, M 86FIR 6) are less well defined. The total integral of the twodimensional Gaussians have been used as a measure of the total fluxes of the sources. For the brighter compact sources, this is in good agreement with simulated aperture photometry, while for the faintest two sources (M 86-FIR 5, M 86-FIR 6), the Gaussians give somewhat higher fluxes because the broad faint wings are also included. However, this is not a major drawback since these sources are anyway extremely close to the detection limit, precluding an accurate photometry. For M 86-FIR 4, only a rough estimate for the brightness at $150 \mu \mathrm{m}$ and $180 \mu \mathrm{m}$ can be derived from Figs. 5 and 7, assuming that a significant fraction of the source structure is already inside the map borders.

The results from the two-dimensional fitting are listed in Table 1, which gives the source designation (Col. 1, see also Fig. 10), the source coordinates (Cols. 2, 3) as derived from the peaks of the Gaussians, the fluxes in $60 \mu \mathrm{m}, 90 \mu \mathrm{m}, 150 \mu \mathrm{m}$, and $180 \mu \mathrm{m}$ bands (Col. 4-7), and finally the suggested optical identification (Col. 8), based on positional associations in the Simbad and NED databases and on optical images (see below). The coordinate for M 86-FIR4 given in Table 1 is the position of the central compact peak, as measured on the one-step drizzled $150 \mu \mathrm{m}$ and $180 \mu \mathrm{m}$ maps (Figs. 5 and 7). A lower limit to its brightness was derived from simple aperture photometry within a rectangular box.

Remarkably, the brighter, eastern component of central double source near M 86 seen at $150 \mu \mathrm{m}$ and $180 \mu \mathrm{m}$ is not coincident with the optical center of the galaxy. Rather, the western component, which is the fainter one at wavelength $\geq 90 \mu \mathrm{m}$, is within about one map pixel $\left(15^{\prime \prime}\right)$ positionally coincident with the optical position of M $86\left(12^{\mathrm{h}} 26^{\mathrm{m}} 11.7^{\mathrm{s}}+12^{\mathrm{d}} 56^{\mathrm{m}} 46^{\mathrm{s}}\right.$, Argyle \& Clements 1990). In view of the large ISOPHOT detector pixel sizes of $45^{\prime \prime}$ at $90 \mu \mathrm{m}$ and $90^{\prime \prime}$ at $150 \mu \mathrm{m}$ and $180 \mu \mathrm{m}$, this positional agreement is excellent, and the western component is therefore identified as the FIR emission of the central region of the elliptical galaxy M 86 itself. The two-dimensional fitting also corroborates the impression from Fig. 4-8 that M 86-SE is somewhat extended roughly along the north-south direction, while M 86-NW is unresolved. Since the central double source (M 86, M 86-SE) was not resolved by IRAS, the fluxes given by Knapp et al. (1989) and White et al. (1991) refer to the combined source (M $86+$ M 86-SE), while M 86-NW is identical to the displaced off-center source of White et al. (1991) and NGC 4402a of Knapp et al. (1989).

The FIR spectra for the three brighter fully covered sources (M 86, M 86-SE, M 86-NW), based on the photometry of the two-dimensional Gaussian fits (Table 1), is shown in Fig. 11. There is a clear difference in the spectral energy distributions (SED) of M 86-NW and the compact double source M 86/M 86-SE. The former has a FIR spectrum constantly declining from the shortest $(60 \mu \mathrm{m})$ to the longest $(180 \mu \mathrm{m})$ wavelength observed, indicating rather warm dust as the source of 
Table 1. FIR sources in the M 86 field.

\begin{tabular}{lccccccr}
\hline \hline Source $^{a}$ & $\alpha_{2000}{ }^{b}$ & $\delta_{2000^{b}}$ & $\begin{array}{l}F_{60 \mu \mathrm{m}} \\
{[\mathrm{mJy}]}\end{array}$ & $\begin{array}{c}F_{90 \mu \mathrm{m}} \\
{[\mathrm{mJy}]}\end{array}$ & $\begin{array}{c}F_{150 \mu \mathrm{m}} \\
{[\mathrm{mJy}]}\end{array}$ & $\begin{array}{c}F_{180 \mu \mathrm{m}} \\
{[\mathrm{mJy}]}\end{array}$ & Suggested Identification \\
\hline \multicolumn{1}{c}{$(1)$} & $(2)$ & $(3)$ & $(4)$ & $(5)$ & $(6)$ & $(7)$ & $(8)$ \\
\hline M 86 & $12^{\mathrm{h}} 26^{\mathrm{m}} 10^{\mathrm{s}}$ & $+12^{\circ} 56^{\prime} 40^{\prime \prime}$ & $\ldots$ & 90 & 220 & 260 & central M 86 dust \\
M 86-SE & $12^{\mathrm{h}} 26^{\mathrm{m}} 14^{\mathrm{s}}$ & $+12^{\circ} 55^{\prime} 30^{\prime \prime}$ & 90 & 170 & 500 & 380 & dust streamers \\
M 86-NW & $12^{\mathrm{h}} 26^{\mathrm{m}} 00^{\mathrm{s}}$ & $+12^{\circ} 59^{\prime} 30^{\prime \prime}$ & 480 & 300 & 220 & 130 & background QSO / ULIRG \\
M 86-FIR4 & $1^{\mathrm{h}} 26^{\mathrm{m}} 02^{\mathrm{s}}$ & $+13^{\circ} 04^{\prime} 55^{\prime \prime}$ & $\ldots$ & $\ldots$ & $>990$ & $>860$ & galaxy VCP 463 \\
M 86-FIR5 $^{d}$ & $12^{\mathrm{h}} 26^{\mathrm{m}} 26^{\mathrm{s}}$ & $+12^{\circ} 58^{\prime} 20^{\prime \prime}$ & $\ldots$ & $(90)$ & $(120)$ & $(100)$ & galaxy VCP 479 \\
M 86-FIR6 $^{d}$ & $12^{\mathrm{h}} 26^{\mathrm{m}} 37^{\mathrm{s}}$ & $+12^{\circ} 56^{\prime} 40^{\prime \prime}$ & $\ldots$ & $(90)$ & $(130)$ & $(100)$ & - \\
M 86-FIR7 $^{d}$ & $12^{\mathrm{h}} 25^{\mathrm{m}} 46^{\mathrm{s}}$ & $+12^{\circ} 55^{\prime} 10^{\prime \prime}$ & $\ldots$ & $\ldots$ & $\ldots$ & $\ldots$ & \\
\hline
\end{tabular}

Notes :

${ }^{a}$ For a schematic overview of the sources in the M 86 field, see Fig. 10.

${ }^{b}$ Positions of 2-D Gaussians fitted to the ISOPHOT maps.

${ }^{c}$ Position of central compact component from $150 \mu \mathrm{m}$ and $180 \mu \mathrm{m}$ maps (Figs. 5 and 7); partial covering precludes accurate estimation total flux and size of surrounding halo.

${ }^{d}$ Positions and fluxes uncertain because sources are close to detection limit.

the FIR emission. The peak of its SED is likely not even within the observed wavelength range, making a derivation of the dust temperature uncertain. In contrast, both M 86 and M 86SE have SEDs strongly increasing with wavelengths, attaining their peaks at much longer wavelengths, indicating dust with a very much lower temperature. The maximum of the M 86-SE SED might be close to $180 \mu \mathrm{m}$ while the SED of M 86 itself still appears to rise even at the longest wavelengths observed $(180 \mu \mathrm{m})$ with a maximum possibly around or even beyond $200 \mu \mathrm{m}$.

A comparison with the $60 \mu \mathrm{m}$ and $100 \mu \mathrm{m}$ fluxes from IRAS given by White et al. (1991) reveals an excellent agreement between the two datasets, given the uncertainties in the ISOPHOT P32 chopped-mode calibration and the differences in the detector band-passes. Except for the $100 \mu \mathrm{m}$ brightness of M 86-NW, a good agreement is also found with the IRAS fluxes derived by Knapp et al. (1989). The $100 \mu \mathrm{m}$ flux of M 86NW listed by Knapp et al. (1989), however, is far too high, thereby incorrectly suggesting that M 86-NW also has a cold SED increasing with wavelength.

Dust temperatures have been derived by fitting a modified blackbody (Planck) function

$F_{v} \propto \nu^{\beta} B_{v}\left(T_{\text {Dust }}\right)$

with an emissivity index $\beta$ to the ISOPHOT fluxes of M 86$\mathrm{NW}$ and the co-added source fluxes of M $86+\mathrm{M}$ 86-SE. The combined source fluxes were used for the latter because the differences between their SEDs are only minor, with the slight flux increase of M 86 beyond $150 \mu \mathrm{m}$ possibly only the result of an insufficient separation of the two components.

Although not immediately obvious from Fig. 11, the declining SED of M 86-NW is rather flat compared to the RayleighJeans part of common dust spectra, and the best fit is actually obtained with a temperature of $T_{\text {Dust }} \approx 100 \mathrm{~K}$ and an emissivity index $\beta \approx 0$. A steeper FIR spectrum with a significantly higher emissivity index can only be obtained if the long wavelengths fluxes from the $\mathrm{C} 200$ detector would be too high by a factor $>2$ relative to the $\mathrm{C} 100$ detector. This, however, is excluded by the overall calibration accuracy $\$ 30 \%$ of the ISOPHOT chopped mode (Laureijs et al. 2000), indicating a very unusual FIR spectrum indeed. Allowing a significant departure of the blackbody fit from the measured fluxes, a lower limit for the dust temperature would be $T_{\text {Dust }} \approx 60 \mathrm{~K}$ with an emissivity index $\beta \approx 2$, i.e. the maximum of the SED would be just short-ward of $60 \mu \mathrm{m}$. Clearly, to tightly constrain the dust temperature of M 86-NW, more data points around the peak of the SED and short-ward thereof are needed.

The SED of the central double source (excluding the $60 \mu \mathrm{m}$ point but including the IRAS $100 \mu \mathrm{m}$ flux from White et al. 1991 ) can be reconciled with a dust temperature $T_{\text {Dust }} \approx 18 \mathrm{~K}$ and an emissivity index $\beta \approx 2$, where the peak of the SED is reached near $200 \mu \mathrm{m}$. This is similar to the average of nonactive spiral galaxies (Stickel et al. 2000), indicating that there is no significant star-forming activity going on in the center of M 86 or in the region where the FIR emission of M 86-SE originates, in accord with the absence of emission line in the optical spectrum (Ho et al. 1995). Again, more data points near the maximum and particularly beyond are needed to get an unambiguous dust temperature, since the SED might be continuously rising even beyond $200 \mu \mathrm{m}$, which would indicate dust temperatures down to $T_{\text {Dust }} \approx 15 \mathrm{~K}$ or even below.

An estimation of the dust mass associated with the FIR sources can be obtained from

$M_{\text {Dust }}=D^{2} F_{v}\left[\kappa_{\lambda} B_{v}\left(T_{\text {Dust }}\right)\right]^{-1}$

(Hildebrand 1983; Draine 1990), where $F_{v}$ is the flux density, D the distance, $\kappa_{\lambda}$ the dust opacity, and $T_{\text {Dust }}$ the dust color temperature. Since dust opacities are rather uncertain, representative values of $3 \mathrm{~m}^{2} / \mathrm{kg}$ and $10 \mathrm{~m}^{2} / \mathrm{kg}$ for $180 \mu \mathrm{m}$ and $60 \mu \mathrm{m}$, respectively, were used, which lie in the middle of the range of currently considered values (Draine 1990)

Assuming that the FIR sources M 86, M 86-SE, and M 86-FIR4 lie at the distance of the elliptical galaxy M 86 (19.5 Mpc, Neilsen \& Tsvetanov 2000), and that the 


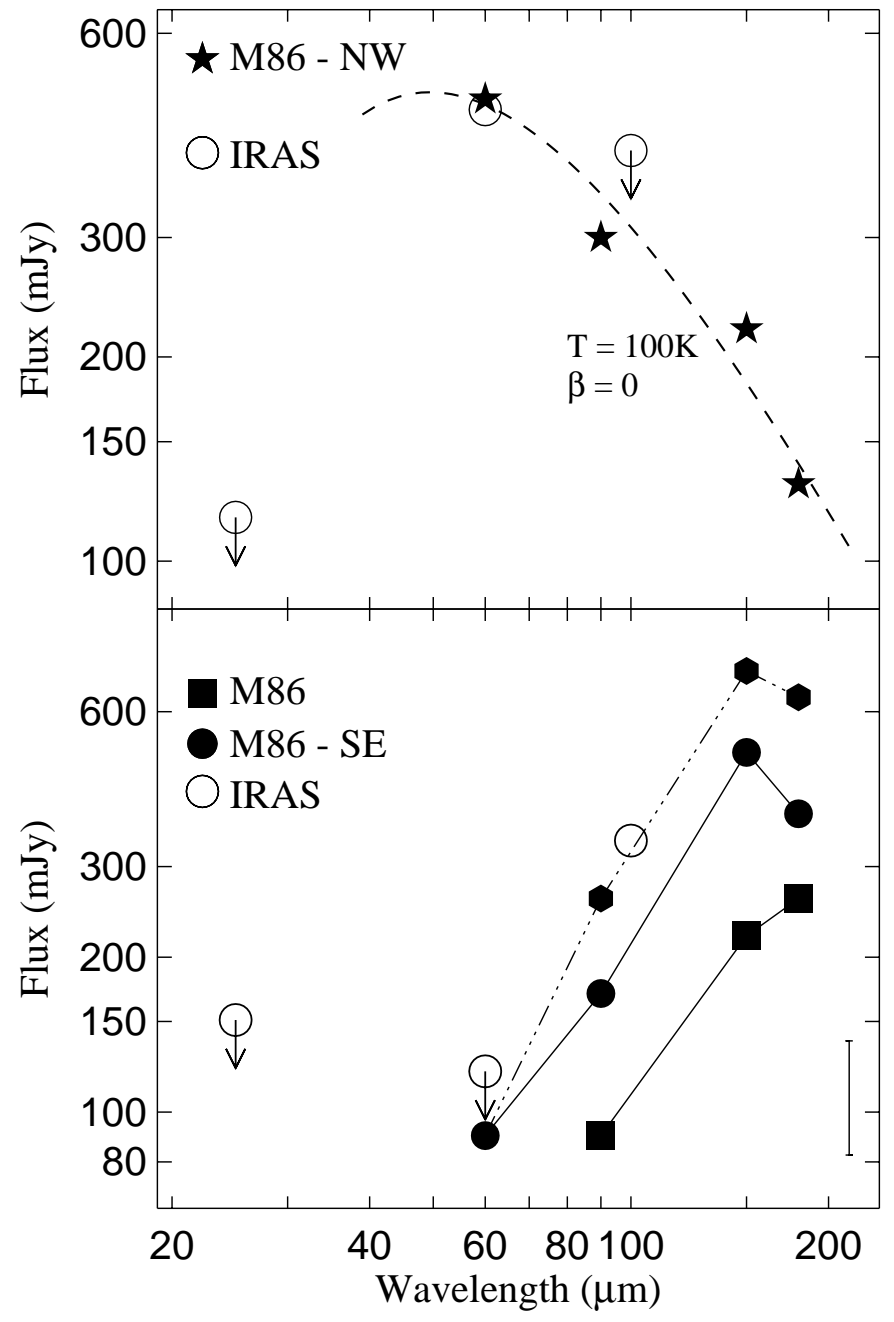

Fig. 11. FIR spectra of the off-center source M 86-NW (top) and the compact double source M 86/M 86-SE (bottom). Filled symbols indicate ISOPHOT fluxes, while IRAS fluxes are represented by open circles (upper limits with downward arrows). For M 86-NW, the dashed curve shows the best fit blackbody spectrum with $T_{\text {Dust }}=100 \mathrm{~K}$ and $\beta=0$. The dot-dashed in the lower panel is the sum of the M 86 and M 86-SE SEDs. The dissimilarity of the SEDs is striking, as is the agreement of the ISOPHOT and IRAS fluxes.

thermal dust emission can be characterized by a dust temperature $T_{\text {Dust }} \approx 18 \mathrm{~K}$, the associated dust masses from the $180 \mu \mathrm{m}$ fluxes are $\approx 5 \times 10^{6} M_{\odot}, \approx 7.5 \times 10^{6} M_{\odot}$, and $\approx 1.5 \times 10^{7} M_{\odot}$, respectively. Due to the continuously rising SED beyond the $100 \mu \mathrm{m}$ IRAS wavelength limit, the combined dust mass of M 86 and M 86 -SE $\left(\approx 1.25 \times 10^{7} M_{\odot}\right)$ is about a factor of 10 larger than that derived from the IRAS data alone (Forbes 1991). Because M 86-NW has a much higher dust temperature as indicated by its decreasing FIR SED, its dust mass would be extremely low $\left(\approx 3.5 \times 10^{3} M_{\odot}\right)$, if it were also located at the distance of M 86. Since it is likely a background source, the associated dust mass depending on the distance may be expressed as $M_{\text {Dust }} \approx 3 \times 10^{5} \times(D / 100 \mathrm{Mpc})^{2} M_{\odot}$, which reaches typical dust masses of $\approx 10^{6} M_{\odot}$ and $\approx 10^{8} M_{\odot}$ only beyond redshifts of $z \approx 0.07$ and $z \approx 0.6$, respectively.

As noted above, all of the seven detected FIR sources are lying within the optical stellar halo of M 86 (Fig. 12). Optical
M86 B-I / 60-180 um contours

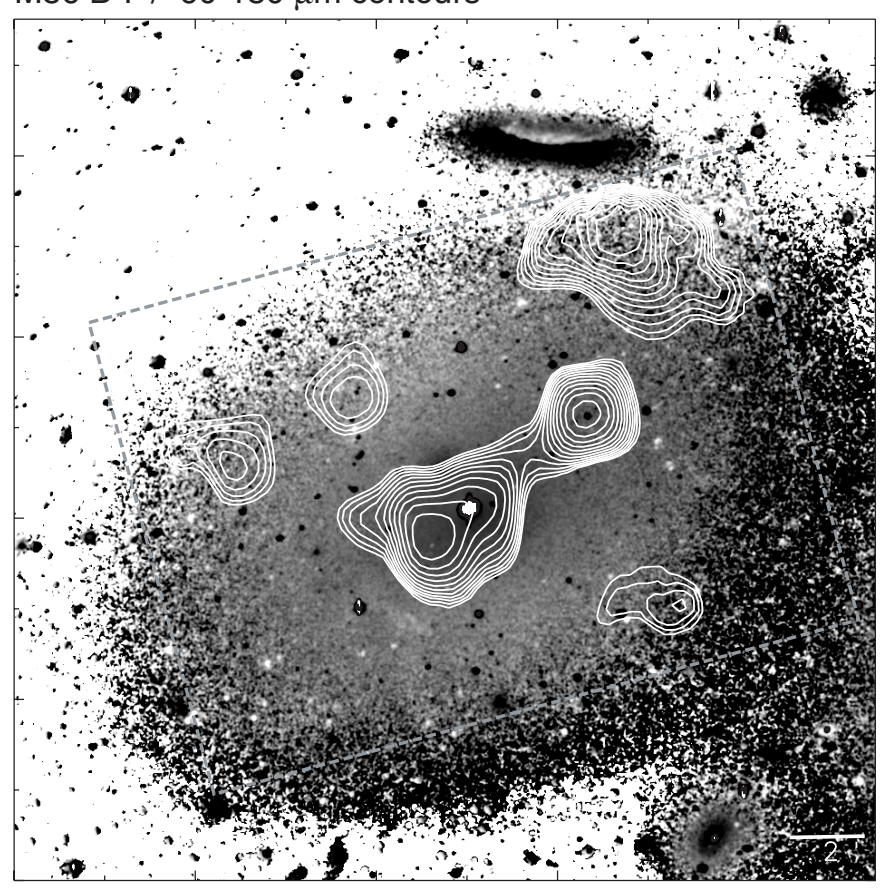

Fig. 12. The ISOPHOT FIR "white light" (60-180 $\mu \mathrm{m})$ image of M 86 (contours) overlaid on a gray-scale representation of the optical $B-I$ color index image. The border of the FIR mapped region is indicated by the dashed rectangle. Image scale is $1.45^{\prime \prime}$ pixel $^{-1}$, the image is rotated clockwise by $\approx 15^{\circ}$ from the standard orientation with north up and east to the left.

counterparts for the FIR sources M 86-NW, M 86-FIR4, and $\mathrm{M}$ 86-SE were searched for on the deep $B$ and $I$ band images published recently by Elmegreen et al. (2000). To get rid of the

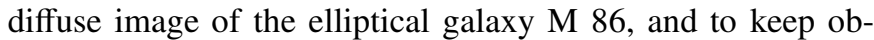
jects up to several times the size of unresolved sources, a twodimensional morphological rolling ball filter (Sternberg 1986) was applied to the optical images and the filtered result subtracted from the original images. The unresolved FIR source M 86-NW is found to be positionally coincident within $\approx 15^{\prime \prime}$ with an unresolved stellar object (Fig. 13), which is therefore proposed as the optical counterpart of M 86-NW (Table 1). In conjunction with its warm FIR spectrum, this suggests that M 86-NW is most likely a background source such as distant starburst or ultra-luminous infrared galaxy or even a quasar, viewed through the foreground stellar halo of M 86. This possibility has already been suggested by W. Rice, as reported by White et al. (1991). It should be noted that, although the FIR position of M 86-NW is close to the IRAS position listed by Leggett et al. (1987), their $B$ band image shows hardly any resemblance to the optical image of the field surrounding M 86NW (Fig. 13).

Although the unusual core-halo sources M 86-FIR 4 is the brightest of the cold FIR sources in the M 86 field, neither the morphologically filtered $B$ and $I$ images of Elmegreen et al. (2000) show a candidate for an optical counterpart, nor shows the $B-I$ color index image (Fig. 12) anything with a color different than the surrounding stellar halo of the elliptical galaxy. The only evidence for something unusual in the 


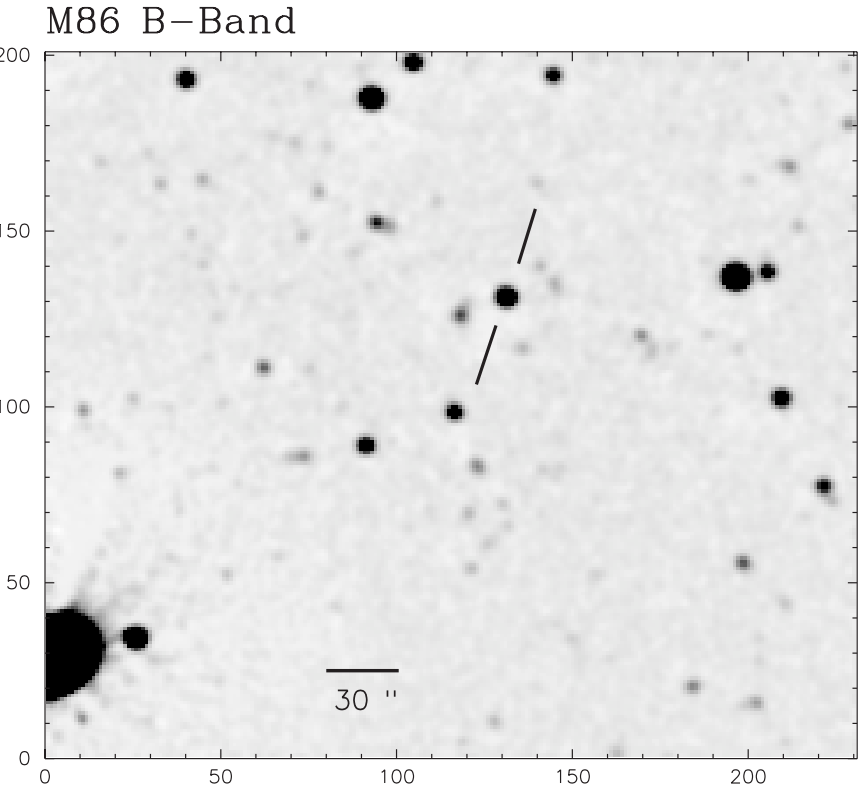

Fig. 13. Residual $B$-band image of the north-western quadrant of $M 86$ after subtraction of a morphological filtered version of the direct image of Elmegreen et al. (2000). The large blob in the lower left corner is the central region of M 86 itself. The optical source nearest to the FIR position of M 86-NW is marked. Image scale is $1.45^{\prime \prime}$ pixel $^{-1}$, north is up and east to the left

optical image near the location of M 86-FIR 4 are the distorted outer isophotes of M 86 elongated towards a direction southwest of the neighboring galaxy NGC 4402 (Nulsen \& Carter 1987; Katsiyannis et al. 1998; Elmegreen et al. 2000). Only a much larger FIR map of the area surrounding M 86 can reveal whether M 86-FIR 4 is actually only the south-eastern region of a much larger dust structure located in the vicinity of or even associated with NGC 4402.

The north-south elongated FIR source M 86-SE south-east of the galaxy center has its peak of the FIR emission close to several faint optical sources, but an optical counterpart similarly extended as the FIR source is not immediately obvious in the $B$ and $I$ images of Elmegreen et al. (2000). However, the dust streamers described by Elmegreen et al. (2000) are approximately at the right distance from the galaxy center and are oriented mainly along the north-south direction, similar to the elongation of M 86-SE. Furthermore, the brightest part of the FIR emission of M 86-SE is towards the southern end, where a large dust patch is located (Fig. 14). This can be taken as evidence that there is in fact no compact optical counterpart for the FIR source M 86-SE, but instead, the FIR emission is due to the thermal emission of the dust trails ascribed to originate in the interacting companion VCC 882 . The $B-I$ color index image (Fig. 14) from the data set of Elmegreen et al. (2000) shows these narrow dust structures quite clearly. In addition, the $B-I$ color index of the M 86 galaxy shows a marked asymmetry, with a much redder color east of the dust streamers compared to the same distance west of the galaxy center. This indicates a break in the large scale $B-I$ color index distribution, which can also be seen in the one-dimensional $B-I$ color index distributions shown by Elmegreen et al. (2000). A possible cause for

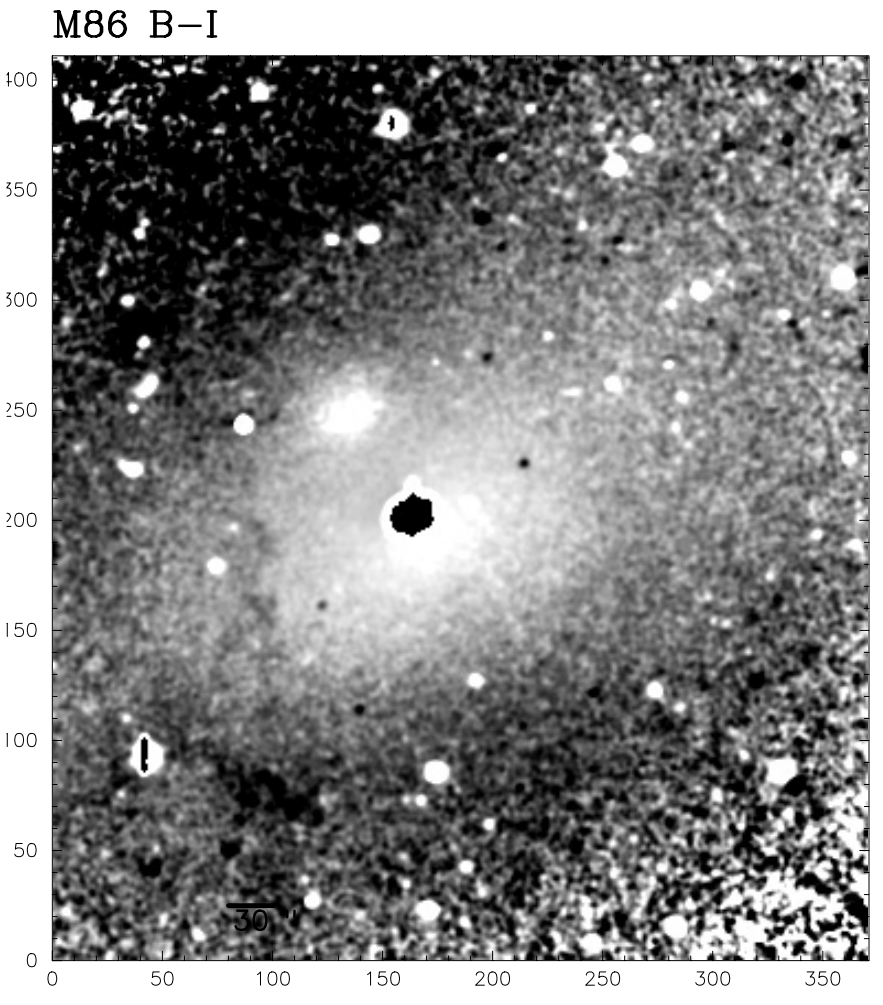

Fig. 14. Gray-scale representation of the $B-I$ color index image of M 86 from the data set of Elmegreen et al. (2000). Redder features appear darker, while bluer color corresponds to lighter gray and white. The north-south oriented dust features east of the center of M 86 appear as black structures silhouetted against the bluer stellar halo of the galaxy. The $B-I$ color index of the galaxy east of the dust features is markedly redder compared to the same distance west of the center. A large dust patch is located at the southern end of narrow dust features, seen as a roughly east-west elongated black structure between the two brighter stars. The $B-I$ color index of the inner part of M 86 shows a marked minor axis asymmetry, redder towards the companion VCC 882 (north-east), and bluer towards the other side. Image scale is $1.45^{\prime \prime}$ pixel $^{-1}$, the image is rotated clockwise by $\approx 15^{\circ}$ from the standard orientation with north up and east to the left.

that could be a foreground dust screen spread out across a significant part of the galaxy affecting the M $86 B-I$ color index beyond $\approx 2^{\prime}$ east of the center.

Although this gives a coherent picture for the FIR sources M 86 and M 86-SE associated with dust within the elliptical galaxy itself, it should be noted that the distance of the peak of M 86-SE from the FIR source M 86 (assumed to be coincident with the center of the optical galaxy) is only $\approx 1.5^{\prime}$, while the distance from the center of the galaxy to the dust streamers in Fig. 14) is $\approx 2^{\prime}$ and to the large blob at the southern end is $\approx 3^{\prime}$. These differences might be due to the crude sampling of very narrow dust features by the rather large ISOPHOT detector pixels (45" at $60 \mu \mathrm{m}$ and $90 \mu \mathrm{m}, 90^{\prime \prime}$ at $150 \mu \mathrm{m}$ and $\left.180 \mu \mathrm{m}\right)$. It can not be completely excluded, however, that both FIR sources M 86/M 86-SE are actually lying roughly symmetrically to the M 86 galaxy center and that M 86-SE is not associated with the dust streamers. Only higher resolution FIR imaging data can resolve this ambiguity. 


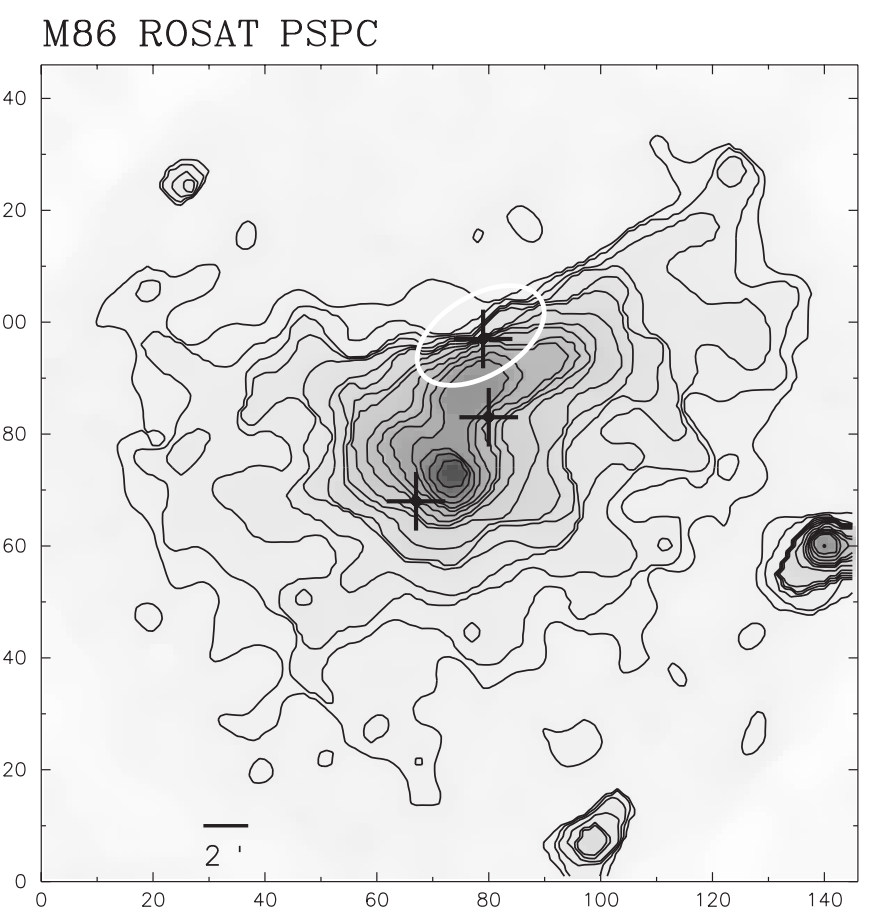

Fig. 15. Adaptively filtered ROSAT PSPC (0.2-2 kev) image of the M 86 field. The positions of the FIR sources M 86-NW, M 86-SE, and M 86-FIR 4 (see Fig. 10 are indicated by crosses. A rough outline of the extended halo of M 86-FIR 4 is indicated by the white ellipse. Image scale is $15^{\prime \prime}$ pixel $^{-1}$, north is up and east to the left.

To check the association of the three FIR sources M 86SE, M 86-NW, and M 86-FIR 4 with the X-ray emission, the ROSAT PSPC (0.2-2 kev) and ASCA GIS (0.7-4 kev) images first published and analyzed by Rangarajan et al. (1995) and Matsushita et al. (1994), respectively, were retrieved from the archives. Figures 15 and 16 show the X-ray images, smoothed with an adaptive filter algorithm (Flaig et al. 1997). The overall morphology of the X-ray emission of the ROSAT and ASCA images is broadly consistent, clearly showing the compact core and the extended, somewhat curved extended emission of the plume. However, the strong cut-off of the X-ray emission $\approx 6^{\prime}$ north of the center seen in the ROSAT image and quite obvious also in the Chandra image of Forman et al. (2001) is much less pronounced in the ASCA image. Furthermore, the large-scale decline in X-ray brightness giving rise to the butterfly-shaped ROSAT X-ray morphology (the northern void, Rangarajan et al. 1995) is also much less conspicuous in the higher energy ASCA image. Thus, only the low energy X-ray morphology appears to be highly asymmetric, while the high energy X-ray structure seems less disturbed. This could be due to low energy absorption, although it can not be completely excluded that it is mainly due to the broad PSF of ASCA. The currently available Chandra imaging data (Forman et al. 2001) are not of sufficient depth to distinguish between these two alternatives (W. Forman, priv. comm.). Further Chandra and $\mathrm{XMM} /$ Newton imaging is expected resolve the issue.

Assuming that, within the uncertainties, the position of M 86 itself is coincident with the peak of the X-ray emission, the positions of the FIR sources were located in the X-ray

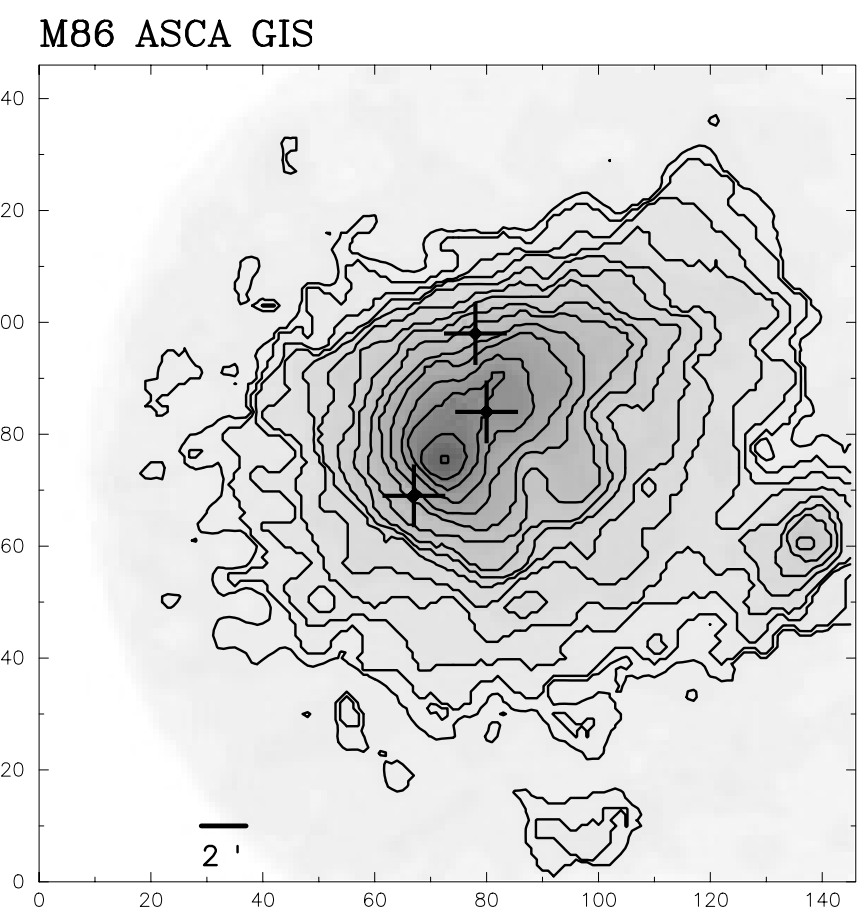

Fig. 16. Adaptively filtered ASCA GIS (0.7-4 kev) image of the M 86 field. The positions of the FIR sources M 86-NW, M 86-SE, and M 86FIR 4 are indicated by crosses. Image scale is $15^{\prime \prime}$ pixel $^{-1}$, north is up and east to the left.

images. In both images, M 86-SE is situated on the steep gradient towards south-east, which is much more pronounced in the ROSAT image. M 86-NW lies definitely away from the ridge of the X-ray plume, also in a region of a rather steep gradient in the ROSAT image. Remarkably, the only partially covered extended FIR source M 86-FIR 4 lies at the position of sharp curved drop of the X-ray emission most clearly seen in the ROSAT image (Fig. 15).

FIR emission directly from the region of ridge of X-ray plume is not detected even in the co-added FIR "white light" image (Fig. 9). The brightness of the FIR sources M 86-FIR 6 clearly detected in all bands can therefore be safely taken as a strict upper limit for the FIR flux from the plume. For a dust temperature of $T_{\text {Dust }} \approx 20 \mathrm{~K}$ expected for dust destroyed in a hot X-ray environment (Dwek et al. 1990), any dust associated with the plume would have a mass well below $\approx 10^{5} M_{\odot}$. This indicates that the plume does not contain any significant amount of dust radiating in the FIR.

\section{Discussion}

The new deep ISOPHOT data revealed the FIR morphology of the M 86 field to be strikingly different from that derived from the lower resolution IRAS data, containing previously unknown cold sources with SEDs increasing beyond $100 \mu \mathrm{m}$. In addition to the previously known off-center source (M 86NW), which is found to be coincident with an unresolved optical source, the FIR emission near the center of the M 86 galaxy is resolved into two components. The western component (M 86) is identified with the FIR emission of the central dust of the M 86 galaxy itself. The other component 
(M 86-SE) does not have an obvious optical counterpart, but it is of similar extent and roughly at the position of optically discovered dust trails attributed to ram stripped ISM from the companion galaxy VCC 882 (Elmegreen et al. 2000). The brightest FIR source M 86-FIR 4 is lying close to the neighboring spiral galaxy NGC 4402, but is also without an optical counterpart.

The dust distribution in M 86 as inferred from the FIR emission bears hardly any resemblance to the HI emission recently mapped with the VLA (Li \& van Gorkom 2001). While the FIR emission is located in the center and south-east of M 86, HI emission strongly peaks south of the center with additional structures east and north-west of the center. The total HI gas mass is $\approx 2.5 \times 10^{8} M_{\odot}$ (Bregman \& Roberts $1990 ; \mathrm{Li}$ \& van Gorkom 2001), while the total dust mass of M 86/M 86$\mathrm{SE}$ is $\approx 1.25 \times 10^{7} M_{\odot}$, giving a gas-to-dust ratio of $\approx 20$, much smaller than the galactic value and that found by Fich \& Hodge (1993) for a number of early-type galaxies, although a few similar low values in elliptical galaxies are known (Henkel \& Wiklind 1997; Oosterloo et al. 2002). The repeated nondetection of M 86 in CO (Lees et al. 1991; Wiklind et al. 1995; Braine et al. 1997) argues against a additional large amount of molecular gas at least near the center, although a full CO map would be needed to check any asymmetric $\mathrm{CO}$ emission and its relation to the detected dust and HI. Furthermore, the absence of detectable HI emission from the brightest FIR source M 86FIR 4 is striking, which could either be due to its radial velocity not covered by the HI observation, or even indicate that this large amount of dust $\approx 1.5 \times 10^{7} M_{\odot}$ is not accompanied by an corresponding amount of $\mathrm{HI}$.

The FIR spectra of the four components are quite different. While the SED of the two components near the M 86 galaxy center (M 86, M 86-SE) and of the source lying towards NGC 4402 (M 86-FIR 4) reach their maxima around or beyond $180 \mu \mathrm{m}$, indicating cold dust with a color temperature in the range $15-20 \mathrm{~K}$, the off-center source $\mathrm{M} 86-\mathrm{NW}$ is much warmer and has a FIR spectrum steeply declining from the shortest wavelengths $(60 \mu \mathrm{m})$ onwards, with a dust temperature $T_{\text {Dust }} \gtrsim 60 \mathrm{~K}$. The central dust structures M 86/M 86-SE have the lowest dust temperatures known in early-type galaxies, even with sub-millimeter data included (Fich \& Hodge 1993; Henkel \& Wiklind 1997). The clear separation of the warm SED of M 86-NW from the other three cold FIR sources is striking evidence for M 86-NW being completely unrelated to M 86, and in particular not associated with the extended X-ray plume. It is most likely a distant luminous infrared source such as a starburst, an ULIRG, or a quasar, and provides no supporting evidence for the ram pressure dust stripping scenario.

There appears to be some confusion about the association of the FIR source M 86-NW with the extended X-ray emission of M 86 (the plume). In the EINSTEIN data, M 86-NW was found to lie close to a small knot south-west of the plume (White et al. 1991). In the original ROSAT HRI and PSPC data analysis (Rangarajan et al. 1995), it was mentioned that, given the uncertainty in the IRAS positions, the off-center FIR source M 86-NW is associated with the plume, although this direct association was not shown. Furthermore, the X-ray knot seen in the EINSTEIN data apparently did not reappear in the
ROSAT data. With the improved positional information from the ISOPHOT maps, it is clear that the FIR source M 86-NW lies away from the ridge of the X-ray plume. Since it is likely a background source, it appears to be only fortuitously located on a steep gradient towards a minimum of the X-ray emission south of the plume (Figs. 15 and 16).

The cold FIR source M 86-SE with its likely origin in the dust streamers from the close companion VCC 882 is also lying on a steep gradient of the ROSAT X-ray emission. Moreover, it is displaced from the M 86 nucleus roughly along the direction of the inferred tangential velocity vector of M 86 (White et al. 1991), which moreover is in obvious contrast to the expectation from the ram pressure picture. The central FIR emission and the associated dust mass of M 86 is similar to the dust masses found from IRAS data in other elliptical galaxies (Bregman et al. 1998), again providing no evidence for ongoing dust stripping. It is likely originating in either the stellar mass loss, or in a merging event in the distant past (Forbes 1991), the latter of which is also indicated in the kinematically separated, rotating core region (Fried \& Illingworth 1994; Arnaboldi et al. 1996). Eventually, neither the nearby companion VCC 882 nor M 86 itself is likely responsible for M 86-FIR 4, since neither seem to have a sufficient amount of ISM to provide the dust seen in the FIR, which again argues against the dust stripping scenario.

The strong depression of the lower energy X-ray emission detected by ROSAT (Rangarajan et al. 1995) and Chandra (Forman et al. 2001) is closely aligned with the extended cold FIR source M 86-FIR 4, whereby its central compact component lies on the steep gradient at the southern end of the hole. The absence of a similar strong gradient in the higher energy ASCA image is taken as evidence that the steep northern decline in X-ray emission is actually due to a foreground X-ray absorber, identified with the FIR source M 86-FIR 4. A rough estimate of the associated column density using the mass of the dust in M 86-FIR $4\left(\approx 1.5 \times 10^{7} M_{\odot}\right)$, its area $\left(\approx 10 \square^{\prime}\right)$, and the standard galactic gas-to-dust ratio $(\approx 200)$ gives $N_{\mathrm{H}} \approx$ $10^{21} \mathrm{~cm}^{-2}$, much higher than the galactic foreground column density (Rangarajan et al. 1995), and consistent with significant low energy X-ray absorption.

This is the first indication that X-ray absorption by foreground dust plays a role in the complex overall X-ray morphology of M 86, which has as yet only been interpreted within the dynamical ram stripping picture due to the fast movement of M 86 across the Virgo cluster. This also nicely confirms the results of the ram pressure stripping simulation of Toniazzo \& Schindler (2001), which required an additional cause for the steep northern X-ray decline. One might further speculate that not only the steep northern X-ray cut-off but additionally much of the butterfly-shaped low-energy X-ray morphology (Fig. 15) is actually caused by large scale absorber spread across an area with $\approx 10^{\prime}$ diameter north of $\mathrm{M} 86$. Whether the required amount of gas of a putative foreground absorber would be larger than the total ISM mass of M 86 (Rangarajan et al. $1995)$ is largely irrelevant if the required gas and dust is actually coming from a nearby spiral galaxy. It should be noted that NGC 4402 is actually roughly centered on this northern X-ray hole. 
Originally, dust scattering had been suggested as the cause for the asymmetric outer isophotes of M 86 (Nulsen \& Carter 1987). Although the ISOPHOT data show M 86-FIR 4 to lie approximately at the position of the distortion, the $B-I$ color index there does not appear to be significantly redder than the rest of the western part of the M 86 galaxy (Fig. 12). This argues against dust scattering as the cause for the distorted outer isophotes of M 86. Yet, the FIR source M 86-FIR 4 appears to be related the strong northern X-ray decline. This can be reconciled if the optical distortion has its origin in gravitational interaction with a neighboring galaxy and that M 86-FIR 4 lies on the far side of the optical M 86 galaxy but still in front of the even more distant extended X-ray emission. Clearly, the X-ray plume as a kind of trail well behind M 86 is consistent with its high radial velocity along the line-of-sight towards the observer.

Although gravitational interaction has hitherto been considered to be less important for the interpretation of the observational data of M 86, the presence of FIR dust emission spread out across the stellar halo of M 86, particularly in the direction of NGC 4402, strongly suggest that tidal interaction is going on within the M 86 galaxy group while entering the Virgo cluster. This is particularly evident in the deep $B$ band image of Arnaboldi et al. (1996), which shows diffuse optical emission surrounding the whole M 86 group and possibly a partial shell structure at the north-western end of the major axis of M 86.

Although the optical stellar halo of M 86 overlaps with that of the nearby elliptical M $84 \approx 17^{\prime}$ west (Arnaboldi et al. 1996; Katsiyannis et al. 1998), the absence of isophotal distortions in M 84 (Arnaboldi et al. 1996) argues against an interaction of M 86 with M 84. Distance measurements based on surface brightness fluctuations also indicate that M 86 is at least $1 \mathrm{Mpc}$ farther away than M 84 (Neilsen \& Tsvetanov 2000). A prime candidate for gravitational interaction clearly is thus the nearest neighboring galaxy NGC 4402, usually classified as of spiral type. In deep images the asymmetric isophotes of M 86 almost reach out to NGC 4402 (Nulsen \& Carter 1987; Katsiyannis et al. 1998; Elmegreen et al. 2000). In optical images NGC 4402 is described to be very dusty (e.g. van den Bergh \& Pierce 1990; Rossa \& Dettmar 2000), its morphology appears to be a warped disk or even a ring-like structure (Binggeli 1999; Elmegreen et al. 2000), which is also very conspicuous in the $B-I$ color index image (Fig. 14). The $\mathrm{H}_{\alpha}$ line emission morphology appears highly disturbed, unlike other edge-on spiral galaxies with extra-planar diffuse ionized gas (Rossa \& Dettmar 2000). Furthermore, NGC 4402 is also known to have a truncated HI profile (Cayatte et al. 1994) and an asymmetric distribution of $\mathrm{HI}$ spread out over a rather large velocity range of almost $400 \mathrm{~km} \mathrm{~s}^{-1}$ (Warmels 1988). These unusual properties can be taken as evidence that NGC 4402 has been disturbed by a gravitational encounter with M 86, but is much less affected by the stripping process due to its own movement through the Virgo cluster. At least the FIR source M 86-FIR 4, which in fact lies only $\approx 2.5^{\prime}$ away from NGC 4402 roughly at the position of the isophotal distortions of M 86 could therefore by due to dust originating in and tidally removed from NGC 4402. M 86-FIR 4 can be considered as the first direct detection of dust located in the intra-cluster medium of a galaxy cluster. Its likely origin in gravitational interaction between cluster galaxies supports the scenario put forward in Stickel et al. $(1998,2002)$ that such features should be detectable in dynamically young clusters.

In summary, the new ISOPHOT data show that M 86 can hardly any longer serve as the example of the dynamic removal of dust (not gas) from a cluster galaxy via ram pressure stripping while moving through the surrounding ICM. In fact, one would expect that such a phenomenon is more easily observed in spiral rather than an elliptical galaxies, which contain a considerably larger amount of dust. As yet there has been no other good candidate galaxy put forward either from the IRAS nor from the ISO data, where the FIR data gives evidence for the dynamical removal of dust. If dust stripping is at all important, it might well be that the process of dust removal via ram pressure stripping is a gradual one, with the additional possibility of dust destruction outside the denser part of the ISM, and particularly in a surrounding hot intra-cluster medium. The whole ongoing process might therefore be almost unobservable directly, and might thus only be inferred from systematic differences in the distribution of dust masses for a very large sample of galaxies.

Acknowledgements. The development and operation of ISOPHOT were supported by MPIA and funds from Deutsches Zentrum für Luftund Raumfahrt (DLR, formerly DARA). The ISOPHOT Data Centre at MPIA is supported by Deutsches Zentrum für Luft- und Raumfahrt (DLR) with funds of Bundesministerium für Bildung und Forschung, grant No. 50 QI0201.

JNB gratefully acknowledges support from NASA.

We are grateful to Dr. Bill Forman for kindly providing us with his M 86 Chandra data.

This research has made use of NASA's Astrophysics Data System Abstract Service, the Simbad Database, operated at CDS, Strasbourg, France, data from the Infrared Processing and Analysis Center (IPAC) and the NASA/IPAC Extragalactic Database (NED), which are operated by the Jet Propulsion Laboratory, California Institute of Technology, under contract with the National Aeronautics and Space Administration.

\section{References}

Arnaboldi, M., Freeman, K. C., Mendez, R. H., et al. 1996, ApJ, 472, 145

Argyle, R. W., \& Clements, E. D. 1990, Observatory 110, 93

Binggeli, B. 1999, in The Radio Galaxy Messier 87, ed. H.-J. Röser, \& K. Meisenheimer (Berlin: Springer), 9

Braine, J., Henkel, C., \& Wiklind, T. 1997, A\&A, 321, 765

van den Bergh, S., \& Pierce, M. J. 1990, ApJ, 364, 444

Bicay, M. D., \& Giovanelli, R. 1987, ApJ, 321, 645

Bregman, J. N., \& Roberts, M. S. 1990, ApJ, 362, 468

Bregman, J. N., Snider, B. A., Grego, L., \& Cox, C. V. 1998, ApJ, 499, 670

Cayatte, V., Kotanyi, C., Balkowski, C., \& van Gorkom, J. H. 1994, AJ, 107, 1003

Contursi, A., Boselli, A., \& Gavazzi, G., et al. 2001, A\&A, 365, 11

Döbereiner, S., Heidt, J., \& Wagner, S. J. 1996, in New Light on Galaxy Evolution, ed. R. Bender, \& R. L. Davies (Dordrecht: Kluwer), IAU Symp., 171, 364

Doyon, R., \& Joseph, R. D. 1989, MNRAS, 239, 347 
Draine, B. T. 1990, in The Interstellar Medium in Galaxies, ed. H. A. Thronson Jr., \& J. M. Schull (Dordrecht: Kluwer), 483

Dwek, E., Rephaeli, Y., \& Mather, J. 1990, ApJ, 350, 104

van Driel, W., \& van Woerden, H. 1989, A\&A, 225, 317

Elmegreen, D. M., Elmegreen, B. G., Chromey, F. R., \& Fine, M. S. 2000, ApJ, 120, 733

Fabbiano, G., Gioia, I. M., \& Trinchieri, G. 1989, ApJ, 347, 127

Faber, S. M., Wegner, G., Burstein, D., et al. 1989, ApJS, 69, 763

Fich, M., \& Hodge, P. 1993, ApJ, 415, 75

Flaig, A., Arce, G. R., \& Barner, K. E. 1997, Proc. International Conference in Acoustics, Speech, and Signal processing (ICASSP) (Munich, Germany)

Forbes, D. A. 1991, MNRAS, 249, 779

Forman, W., Schwarz, J., Jones, C., Liller, W., \& Fabian, A. C. 1979, ApJ, 234, L27

Forman, W., Markevitch, M., Jones, C., et al. 2001, in Clusters of Galaxies and the High Redshift Universe Observed in X-Rays, Proc. XXI Moriond Conference, ed. D. M. Neumann, \& J. Tranh Thanh Van [astro-ph/0110087]

Fried, J. W., \& Illingworth, G. D. 1994, ApJ, 107, 992

Gabriel, C., Acosta-Pulido, J., Heinrichsen, I., et al. 1997, Astronomical Data Analysis Software and Systems VI, ed. G. Hunt, \& H. E. Payne, ASP Conf. Ser., 125, 108

Giovanelli, R., \& Haynes, M. P. 1983, AJ, 88, 881

Henkel, C., \& Wiklind, T. 1997, Space Sci. Rev. 81, 1

Hildebrand, R. H. 1983, QJRAS, 24, 267

Ho, L. C., Filippenko, A. V., \& Sargent, W. L. W. 1995, ApJS, 98, 477

Hook, R. N., \& Fruchter, A. S. 1997, Astronomical Data Analysis Software and Systems VI, ed. G. Hunt, \& H. E. Payne, ASP Conf. Ser., 125,147

Irwin, J. A., \& Sarazin, C. L. 1996, ApJ, 471, 683

Kalluri, S., \& Arce, G. R. 1998, IEEE Trans. on Signal Proc., 46, 322

Kessler, M. F., Steinz, J. A., Anderegg, M. E., et al. 1996, A\&A, 315, L27

Knapp, G. R., Guhathakurta, P., Kim, D.-W., \& Jura, M. 1989, ApJS, 70, 329

Katsiyannis, A. C., Kemp, S. N., Berry, D. S., \& Meaburn, J. 1998, A\&AS, 132, 387
Laureijs, R. J., Klaas, U., Richards, P. J., Schulz, B., \& Ábrahám, P. 2000, The ISO Handbook, vol. V: PHT - The Imaging PhotoPolarimeter, version 1.1, SAI/99-069/dc

Lees, J. F., Knapp, G. R., Rupen, M. P., \& Phillips, T. G. 1991, ApJ, 379, 177

Leggett, S. K., Clowes, R. G., Kalafi, M., et al. 1987, MNRAS, 227, 563

Lemke, D., Abolins, J., Abraham, P., et al. 1996, A\&A, 315, L64

Lemke, D., \& Klaas, U. 1999, in The Universe as seen by ISO, ed. P. Cox, \& M. F. Kessler, ESA-SP 427, 55

Li, Y., \& van Gorkom, J. 2001, Gas and Galaxy Evolution, ed. J. E. Hibbard, M. P. Rupe, \& J. H. van Gorkom, ASP Conf. Ser., 240, 637

Matsushita, K., Makishima, K., Awaki, H., et al. 1994, ApJ, 436, L41

Michard, R. 1999, A\&A, 137, 245

Neilsen, E. H., \& Tsvetanov, Z. I. 2000, ApJ, 536, 255

Nulsen, P. E. J., \& Carter, D. 1987, MNRAS, 225, 939

Oosterloo, T. A., Morganti, R., Sadler, E. M., Vergani, D., \& Caldwell, N. 2002, AJ, 123, 729

Rangarajan, F. V. N., White, D. A., Ebeling, H., \& Fabian, A. C. 1995, MNRAS, 277, 1047

Rossa, J., \& Dettmar, R.-J. 2000, A\&A, 359, 433

Schindler, S., Binggeli, B., \& Böhringer, H. 1999, A\&A, 343, 420

Solanes, J. M., Manrique, A., Garcia-Gomez, C., et al. 2001, ApJ, 548, 97

Sternberg, S. 1986, Computer Vision, Graphics and Image Proc. 35, 333

Stickel, M., Lemke, D., Mattila, K., et al. 1998, A\&A, 329, 55

Stickel, M., Lemke, D., Klaas, U., et al. 2000, A\&A, 359, 865

Stickel, M., Klaas, U., Lemke, D., \& Mattila, K. 2002, A\&A, 383, 367

Tomita, A., Aoki, K., Watanabe, M., et al. 2000, AJ, 120, 123

Toniazzo, T., \& Schindler, S., 2001, MNRAS, 325, 509

Tran, H. D., Tsvetanov, Z., Ford, H. C., et al. 2001, AJ, 121, 2928

Veron-Cetty, M.-P., \& Veron, P. 1988, A\&A, 204, 28

Warmels, R. H. 1988, A\&AS, 72, 57

White, D. A., Fabian, A. C., Forman, W., Jones, C., \& Stern, C. 1991, ApJ, 375, 35

Wiklind, T., Combes, F., \& Henkel, C. 1995, A\&A, 297, 643 